\title{
Association of Baseline HbA1c with Cardiovascular and Renal Outcomes \\ Analyses from DECLARE-TIMI 58
}

\section{Online only supplementary material}

Supplementary Table 1 - Baseline characteristics - overall population

Supplementary Table 2 - Baseline characteristics - ASCVD population

Supplementary Table 3 - Baseline characteristics - MRF population

Supplementary figure $1-$ Cardiovascular outcomes with dapagliflozin vs. placebo

Supplementary figure 2 - Cardiovascular and renal outcomes with dapagliflozin vs. placebo in the ASCVD population

Supplementary figure 3 - Cardiovascular and renal outcomes with dapagliflozin vs. placebo in the MRF population

Supplementary figure 4 - HbA1c changes with dapagliflozin vs. placebo by baseline HbA1c category 
Supplementary Table 1 - Baseline characteristics - overall population

\begin{tabular}{|c|c|c|c|c|c|}
\hline & $<7 \%$ & $7-<8 \%$ & $8-<9 \%$ & $>=9 \%$ & P-trend \\
\hline & $N=1547$ & $N=6626$ & $N=4520$ & $N=4461$ & \\
\hline $\begin{array}{l}\text { Age, years, median } \\
\text { (IQR) }\end{array}$ & $66.0(61.0-70.0)$ & $65.0(60.0-69.0)$ & $64.0(60.0-68.0)$ & $62.0(58.0-66.0)$ & $<0.0001$ \\
\hline Female sex, $\mathrm{N}(\%)$ & $542(35.0)$ & $2393(36.1)$ & $1725(38.2)$ & $1759(39.4)$ & $<0.0001$ \\
\hline $\begin{array}{l}\mathrm{BMI}, \mathrm{kg} / \mathrm{m}^{2}, \text { median } \\
(\mathrm{IQR}), \mathrm{N}\end{array}$ & 31.5 (28.1-35.3), 1544 & $31.2(27.7-35.2), 6624$ & $31.4(27.9-35.5), 4518$ & $31.3(27.7-35.7), 4459$ & 0.7928 \\
\hline $\begin{array}{l}\text { Diabetes duration, } \\
\text { years, median (IQR), N }\end{array}$ & $9.0(5.0-14.0)$ & $10.0(6.0-16.0), 6625$ & $11.0(7.0-17.0)$ & $11.0(7.0-17.0), 4460$ & $<0.0001$ \\
\hline $\begin{array}{l}\text { Risk Category - ASCVD, } \\
\mathrm{N}(\%)\end{array}$ & $653(42.2)$ & 2619 (39.5) & 1784 (39.5) & $1918(43.0)$ & 0.0330 \\
\hline $\begin{array}{l}\text { History of heart failure, } \\
\mathrm{N}(\%)\end{array}$ & $127(8.2)$ & $608(9.2)$ & 446 (9.9) & $543(12.2)$ & $<0.0001$ \\
\hline $\begin{array}{l}\text { Systolic BP, mmHg, } \\
\text { median (IQR) }\end{array}$ & $134.0(123.5,144.5)$ & $135.0(124.5,145.0)$ & $135.0(124.0,145.0)$ & $135.0(124.0,145.0)$ & 0.8696 \\
\hline Current smoker & $188(12.2)$ & 918 (13.9) & $663(14.7)$ & $729(16.3)$ & $<0.0001$ \\
\hline $\begin{array}{l}\text { Total Cholesterol, } \\
\text { median, (IQR), N }\end{array}$ & $\begin{array}{l}156.0(134.0-183.0), \\
1529\end{array}$ & $\begin{array}{l}160.0(136.0-189.0), \\
6552\end{array}$ & $\begin{array}{l}165.0(138.0-196.0), \\
4476\end{array}$ & $\begin{array}{l}171.0(143.0-205.0), \\
4411\end{array}$ & $<0.0001$ \\
\hline $\begin{array}{l}\text { eGFR, median (IQR), } \\
\mathrm{ml} / \mathrm{min} / 1.73 \mathrm{~m}^{2}, \mathrm{~N}\end{array}$ & $84.0(71.0-93.0)$ & $87.0(73.0-95.0)$ & $89.0(76.0-97.0)$ & 92.0 (78.0-99.0), 4460 & $<0.0001$ \\
\hline $\begin{array}{l}\text { eGFR, }<60 \\
\mathrm{ml} / \mathrm{min} / 1.73 \mathrm{~m}^{2}, \mathrm{~N}(\%)\end{array}$ & $119(7.7)$ & $503(7.6)$ & $325(7.2)$ & $317 / 4460(7.1)$ & 0.2657 \\
\hline $\begin{array}{l}\text { eGFR, } 60-90 \\
\mathrm{ml} / \mathrm{min} / 1.73 \mathrm{~m}^{2}, \mathrm{~N}(\%)\end{array}$ & $876(56.6)$ & 3240 (48.9) & 1984 (43.9) & $1630 / 4460(36.5)$ & $<.0001$ \\
\hline $\begin{array}{l}\text { eGFR, } \geq 90 \\
\mathrm{ml} / \mathrm{min} / 1.73 \mathrm{~m}^{2}, \mathrm{~N}(\%)\end{array}$ & $552(35.7)$ & $2883(43.5)$ & 2211 (48.9) & $2513 / 4460(56.3)$ & $<.0001$ \\
\hline Insulin, N (\%) & $329(21.3)$ & $2256(34.0)$ & $2079(46.0)$ & $2345(52.6)$ & $<0.0001$ \\
\hline Metformin, N (\%) & $1294(83.6)$ & 5495 (82.9) & $3726(82.4)$ & $3548(79.5)$ & $<0.0001$ \\
\hline Sulfonylurea, N (\%) & $531(34.3)$ & $2761(41.7)$ & $2060(45.6)$ & $1968(44.1)$ & $<0.0001$ \\
\hline DPP-4 Inhibitors, N (\%) & $282(18.2)$ & $1179(17.8)$ & $780(17.3)$ & $646(14.5)$ & $<0.0001$ \\
\hline Beta blockers, N (\%) & $845(54.6)$ & $3501(52.8)$ & $2352(52.0)$ & $2330(52.2)$ & 0.1357 \\
\hline ACEI/ARB, N (\%) & 1262 (81.6) & $5488(82.8)$ & 3672 (81.2) & 3526 (79.0) & $<0.0001$ \\
\hline Statin, N (\%) & $1241(80.2)$ & $5042(76.1)$ & $3323(73.5)$ & $3147(70.5)$ & $<0.0001$ \\
\hline Anti-platelet, N (\%) & $988(63.9)$ & $4064(61.3)$ & 2752 (60.9) & $2679(60.1)$ & 0.0159 \\
\hline
\end{tabular}

Legend - ASCVD - Atherosclerotic cardiovascular disease, BP - Blood pressure 
Supplementary Table 2 - Baseline characteristics - ASCVD population

\begin{tabular}{|c|c|c|c|c|c|}
\hline & $<7 \%$ & $7-<8 \%$ & $8-<9 \%$ & $>=9 \%$ & P-trend \\
\hline & $N=653$ & $N=2619$ & $N=1784$ & $N=1918$ & \\
\hline $\begin{array}{l}\text { Age, years, median } \\
\text { (IQR) }\end{array}$ & $65.0(60.0,70.0)$ & $64.0(58.0,69.0)$ & $63.0(58.0,68.0)$ & $61.0(55.0,66.0)$ & $<.0001$ \\
\hline Female sex, $\mathrm{N}(\%)$ & $151(23.1)$ & $693(26.5)$ & $512(28.7)$ & $593(30.9)$ & $<.0001$ \\
\hline $\begin{array}{l}\text { BMI, } \mathrm{kg} / \mathrm{m}^{2}, \text { median } \\
(\mathrm{IQR}), \mathrm{N}\end{array}$ & $31.3(28.0,35.2), 650$ & $\begin{array}{l}31.0(27.7,35.0), \\
2617\end{array}$ & $\begin{array}{l}31.3(28.0,35.5), \\
1783\end{array}$ & $\begin{array}{l}31.7 /(28.1,36.0), \\
1917\end{array}$ & 0.0008 \\
\hline $\begin{array}{l}\text { Diabetes duration, } \\
\text { years, median (IQR), N }\end{array}$ & $9.0(5.0,15.0)$ & $10.0(5.0,16.0), 2618$ & $11.0(6.0,18.0)$ & $11.0(6.0,17.0), 1917$ & $<.0001$ \\
\hline $\begin{array}{l}\text { History of heart failure, } \\
\mathrm{N}(\%)\end{array}$ & $87(13.3)$ & $403(15.4)$ & $292(16.4)$ & $374(19.5)$ & $<.0001$ \\
\hline $\begin{array}{l}\text { Systolic } \mathrm{BP}, \mathrm{mmHg}, \\
\text { median (IQR) }\end{array}$ & $134.0(122.5,145.0)$ & $134.5(124.0,145.5)$ & $132.8(122.5,144.0)$ & $132.5(122.5,143.5)$ & 0.0284 \\
\hline Current smoker & $76(11.6)$ & 375 (14.3) & $264(14.8)$ & 319 (16.6) & 0.0019 \\
\hline $\begin{array}{l}\text { Total Cholesterol, } \\
\text { median, (IQR), N }\end{array}$ & $\begin{array}{l}148.0(127.0,175.0), \\
641\end{array}$ & $\begin{array}{l}151.0(129.0,180.0), \\
2586\end{array}$ & $\begin{array}{l}155.0(129.0,187.0), \\
1767\end{array}$ & $\begin{array}{l}162.0(136.0,196.0), \\
1894\end{array}$ & $<.0001$ \\
\hline $\begin{array}{l}\text { eGFR, median (IQR), } \\
\mathrm{ml} / \mathrm{min} / 1.73 \mathrm{~m}^{2}, \mathrm{~N}\end{array}$ & $84.0(70.0,93.0)$ & $86.0(72.0,95.0)$ & $88.0(73.0,97.0)$ & $91.0(76.0,100.0)$ & $<.0001$ \\
\hline $\begin{array}{l}\text { eGFR, }<60 \\
\mathrm{ml} / \mathrm{min} / 1.73 \mathrm{~m}^{2}, \mathrm{~N}(\%)\end{array}$ & $57(8.7)$ & 258 (9.9) & $160(9.0)$ & $168(8.8)$ & 0.4189 \\
\hline $\begin{array}{l}\text { eGFR, } 60-90 \\
\mathrm{ml} / \mathrm{min} / 1.73 \mathrm{~m}^{2}, \mathrm{~N}(\%)\end{array}$ & $364(55.7)$ & $1250(47.7)$ & $802(45.0)$ & $722(37.6)$ & $<.0001$ \\
\hline $\begin{array}{l}\text { eGFR, } \geq 90 \\
\mathrm{ml} / \mathrm{min} / 1.73 \mathrm{~m}^{2}, \mathrm{~N}(\%)\end{array}$ & $232(35.5)$ & $1111(42.4)$ & $822(46.1)$ & $1028(53.6)$ & $<.0001$ \\
\hline Insulin, N (\%) & 156 (23.9) & $987(37.7)$ & $923(51.7)$ & $1126(58.7)$ & $<.0001$ \\
\hline Metformin, N (\%) & $543(83.2)$ & 2086 (79.6) & $1391(78.0)$ & $1424(74.2)$ & $<.0001$ \\
\hline Sulfonylurea, N (\%) & $222(34.0)$ & $1026(39.2)$ & 747 (41.9) & 766 (39.9) & 0.0223 \\
\hline DPP-4 Inhibitors, N (\%) & $124(19.0)$ & $421(16.1)$ & $307(17.2)$ & 266 (13.9) & 0.0063 \\
\hline Beta blockers, N(\%) & $498(76.3)$ & $1887(72.1)$ & 1282 (71.9) & $1402(73.1)$ & 0.5383 \\
\hline ACEI/ARB, N (\%) & 535 (81.9) & $2189(83.6)$ & $1465(82.1)$ & $1546(80.6)$ & 0.0435 \\
\hline Statin, N (\%) & $582(89.1)$ & $2279(87.0)$ & $1526(85.5)$ & $1620(84.5)$ & 0.0008 \\
\hline Anti-platelet, N (\%) & $580(88.8)$ & 2285 (87.2) & $1536(86.1)$ & $1634(85.2)$ & 0.0073 \\
\hline
\end{tabular}

Legend - ASCVD - Atherosclerotic cardiovascular disease, BP - Blood pressure 
Supplementary Table 3 - Baseline characteristics - MRF population

\begin{tabular}{|c|c|c|c|c|c|}
\hline & $<7 \%$ & $7-<8 \%$ & $8-<9 \%$ & $>=9 \%$ & P-trend \\
\hline & $N=894$ & $N=4007$ & $N=2376$ & $N=2543$ & \\
\hline $\begin{array}{l}\text { Age, years, median } \\
\text { (IQR) }\end{array}$ & $66.0(62.0,70.0)$ & $65.0(61.0,69.0)$ & $64.0(61.0,68.0)$ & $63.0(60.0,67.0)$ & $<.0001$ \\
\hline Female sex, $\mathrm{N}(\%)$ & 391 (43.7) & $1700(42.4)$ & $1213(44.3)$ & 1166 (45.9) & 0.0180 \\
\hline $\begin{array}{l}\text { BMI, } \mathrm{kg} / \mathrm{m}^{2}, \text { median } \\
(\mathrm{IQR}), \mathrm{N}\end{array}$ & $31.7(28.2,35.6)$ & $31.3(27.8,35.3)$ & $\begin{array}{l}31.5(27.9,35.6), \\
2735\end{array}$ & $\begin{array}{l}31.0(27.5,35.4), \\
2542\end{array}$ & 0.0139 \\
\hline $\begin{array}{l}\text { Diabetes duration, } \\
\text { years, median (IQR), N }\end{array}$ & $8.0(5.0,13.0)$ & $10.0(6.0,15.0)$ & $11.0(7.0,17.0)$ & $11.0(7.0,17.0)$ & $<.0001$ \\
\hline $\begin{array}{l}\text { History of heart failure, } \\
\mathrm{N}(\%)\end{array}$ & $40(4.5)$ & $205(5.1)$ & $154(5.6)$ & $169(6.6)$ & 0.0028 \\
\hline $\begin{array}{l}\text { Systolic } \mathrm{BP}, \mathrm{mmHg}, \\
\text { median (IQR) }\end{array}$ & $133.8(124.5,144.0)$ & $135.0(125.0,145.0)$ & $136.0(125.3,145.5)$ & $136.0(125.0,145.0)$ & 0.0203 \\
\hline Current smoker & $112(12.5)$ & $543(13.6)$ & $399(14.6)$ & $410(16.1)$ & 0.0009 \\
\hline $\begin{array}{l}\text { Total Cholesterol, } \\
\text { median, (IQR) }\end{array}$ & $\begin{array}{l}162.0(140.0,188.0), \\
888\end{array}$ & $\begin{array}{l}166.0(142.0,194.0), \\
3966\end{array}$ & $\begin{array}{l}171.0(144.0,200.0), \\
2709\end{array}$ & $\begin{array}{l}176.0(149.0,210.0), \\
2517\end{array}$ & $<.0001$ \\
\hline $\begin{array}{l}\text { eGFR, median (IQR), } \\
\mathrm{ml} / \mathrm{min} / 1.73 \mathrm{~m}^{2}, \mathrm{~N}\end{array}$ & $84.0(72.0,93.0)$ & $87.0(75.0,95.0)$ & $90.0(77.0,97.0)$ & $\begin{array}{l}92.5(80.0,98.0), \\
2542\end{array}$ & $<.0001$ \\
\hline $\begin{array}{l}\text { eGFR, }<60 \\
\mathrm{ml} / \mathrm{min} / 1.73 \mathrm{~m}^{2}, \mathrm{~N}(\%)\end{array}$ & $62(6.9)$ & $245(6.1)$ & $165(6.0)$ & 149 (5.9), 2542 & 0.3451 \\
\hline $\begin{array}{l}\text { eGFR, } 60-90 \\
\mathrm{ml} / \mathrm{min} / 1.73 \mathrm{~m}^{2}, \mathrm{~N}(\%)\end{array}$ & $512(57.3)$ & $1990(49.7)$ & $1182(43.2)$ & 908 (35.7), 2542 & $<.0001$ \\
\hline $\begin{array}{l}\text { eGFR, } \geq 90 \\
\mathrm{ml} / \mathrm{min} / 1.73 \mathrm{~m}^{2}, \mathrm{~N}(\%)\end{array}$ & $320(35.8)$ & $1772(44.2)$ & 1389 (50.8) & 1485 (58.4), 2542 & $<.0001$ \\
\hline Insulin, $\mathrm{N}(\%)$ & $173(19.4)$ & $1269(31.7)$ & $1156(42.3)$ & 1219 (47.9) & $<.0001$ \\
\hline Metformin, N (\%) & $751(84.0)$ & $3409(85.1)$ & $2335(85.3)$ & 2124 (83.5) & 0.3271 \\
\hline Sulfonylurea, N (\%) & $309(34.6)$ & 1735 (43.3) & $1313(48.0)$ & $1202(47.3)$ & $<.0001$ \\
\hline DPP-4 Inhibitors, N (\%) & $158(17.7)$ & 758 (18.9) & $473(17.3)$ & 380 (14.9) & 0.0004 \\
\hline Beta blockers, N (\%) & $347(38.8)$ & $1614(40.3)$ & $1070(39.1)$ & $928(36.5)$ & 0.0131 \\
\hline ACEI/ARB, N (\%) & $727(81.3)$ & $3299(82.3)$ & 2207 (80.7) & 1980 (77.9) & $<.0001$ \\
\hline Statin, N (\%) & $659(73.7)$ & $2763(69.0)$ & $1797(65.7)$ & $1527(60.0)$ & $<.0001$ \\
\hline Anti-platelet, N (\%) & $408(45.6)$ & $1779(44.4)$ & $1216(44.4)$ & 1045 (41.1) & 0.0063 \\
\hline
\end{tabular}

Legend - ASCVD - Atherosclerotic cardiovascular disease, BP - Blood pressure 


\section{Supplementary figure 1}

\section{Cardiovascular outcomes with dapagliflozin vs. placebo}

\begin{tabular}{|c|c|c|c|c|c|c|}
\hline CVD & $\begin{array}{l}\text { Dapagliflozin } \\
\text { n/N (\%) }\end{array}$ & $\begin{array}{l}\text { Placebo } \\
\mathrm{n} / \mathrm{N}(\%)\end{array}$ & & & Hazard Ratio & $\begin{array}{l}\text { Interaction } \\
\text { p-value }\end{array}$ \\
\hline Overall & $245 / 8582(2.9 \%)$ & $249 / 8578$ (2.9\%) & $r-1$ & & $0.98(0.82-1.17)$ & \\
\hline$<7 \%$ & $16 / 773(2.1 \%)$ & $15 / 774(1.9 \%)$ & $\longmapsto$ & $\longrightarrow$ & $1.08(0.53-2.19)$ & 0.8938 \\
\hline $7-<8 \%$ & $81 / 3317(2.4 \%)$ & $90 / 3309(2.7 \%)$ & $\mapsto$ & & $0.90(0.67-1.21)$ & \\
\hline $8-<9 \%$ & $59 / 2193(2.7 \%)$ & $60 / 2327(2.6 \%)$ & $\longmapsto$ & & $1.07(0.75-1.53)$ & \\
\hline$\geq 9 \%$ & $89 / 2297(3.9 \%)$ & $84 / 2164(3.9 \%)$ & $\mapsto$ & & $0.98(0.72-1.32)$ & \\
\hline \multicolumn{7}{|c|}{ Myocardial Infarction } \\
\hline Overall & $393 / 8582(4.6 \%)$ & $441 / 8578(5.1 \%)$ & - & & $0.89(0.77-1.01)$ & \\
\hline$<7 \%$ & $31 / 773(4.0 \%)$ & $36 / 774(4.7 \%)$ & $\longmapsto$ & & $0.85(0.52-1.37)$ & 0.8462 \\
\hline $7-<8 \%$ & $142 / 3317(4.3 \%)$ & $161 / 3309(4.9 \%)$ & $\mapsto$ & & $0.88(0.70-1.10)$ & \\
\hline $8-<9 \%$ & $99 / 2193(4.5 \%)$ & $131 / 2327(5.6 \%)$ & $\mapsto$ & & $0.82(0.63-1.07)$ & \\
\hline$\geq 9 \%$ & $121 / 2297(5.3 \%)$ & $113 / 2164(5.2 \%)$ & $\mapsto$ & & $0.98(0.76-1.26)$ & \\
\hline \multicolumn{7}{|c|}{ Ischemic Stroke } \\
\hline Overall & $235 / 8582(2.7 \%)$ & $231 / 8578(2.7 \%)$ & 1 & & $1.01(0.84-1.21)$ & \\
\hline$<7 \%$ & $21 / 773(2.7 \%)$ & $15 / 774(1.9 \%)$ & $\longmapsto$ & $=$ & $1.34(0.69-2.61)$ & 0.6919 \\
\hline $7-<8 \%$ & $86 / 3317(2.6 \%)$ & $86 / 3309(2.6 \%)$ & $\longmapsto$ & & $1.00(0.74-1.34)$ & \\
\hline $8-<9 \%$ & $59 / 2193(2.7 \%)$ & $71 / 2327(3.1 \%)$ & $\longmapsto$ & & $0.89(0.63-1.25)$ & \\
\hline \multirow[t]{2}{*}{$\geq 9 \%$} & $69 / 2297(3.0 \%)$ & $59 / 2164(2.7 \%)$ & $\mapsto$ & & $1.09(0.77-1.55)$ & \\
\hline & \multicolumn{6}{|c|}{$\begin{array}{l}\quad 2.5 \\
\text {--- Favors placebo ---> }\end{array}$} \\
\hline
\end{tabular}

Legend: CVD - Cardiovascular death 


\section{Supplementary figure 2}

Cardiovascular and renal outcomes with dapagliflozin vs. placebo in the ASCVD population

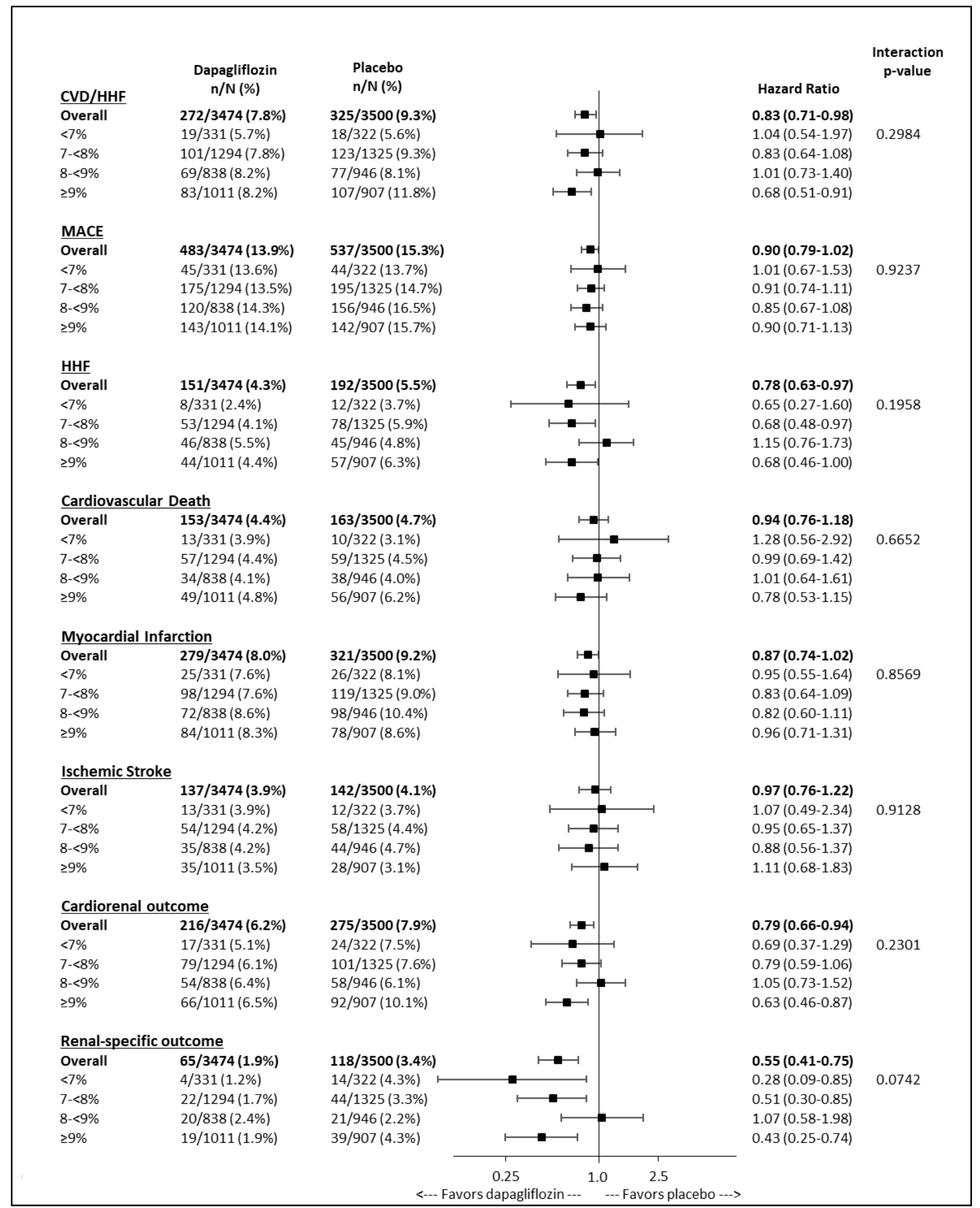




\section{Supplementary figure 3}

Cardiovascular and renal outcomes with dapagliflozin vs. placebo in the MRF population

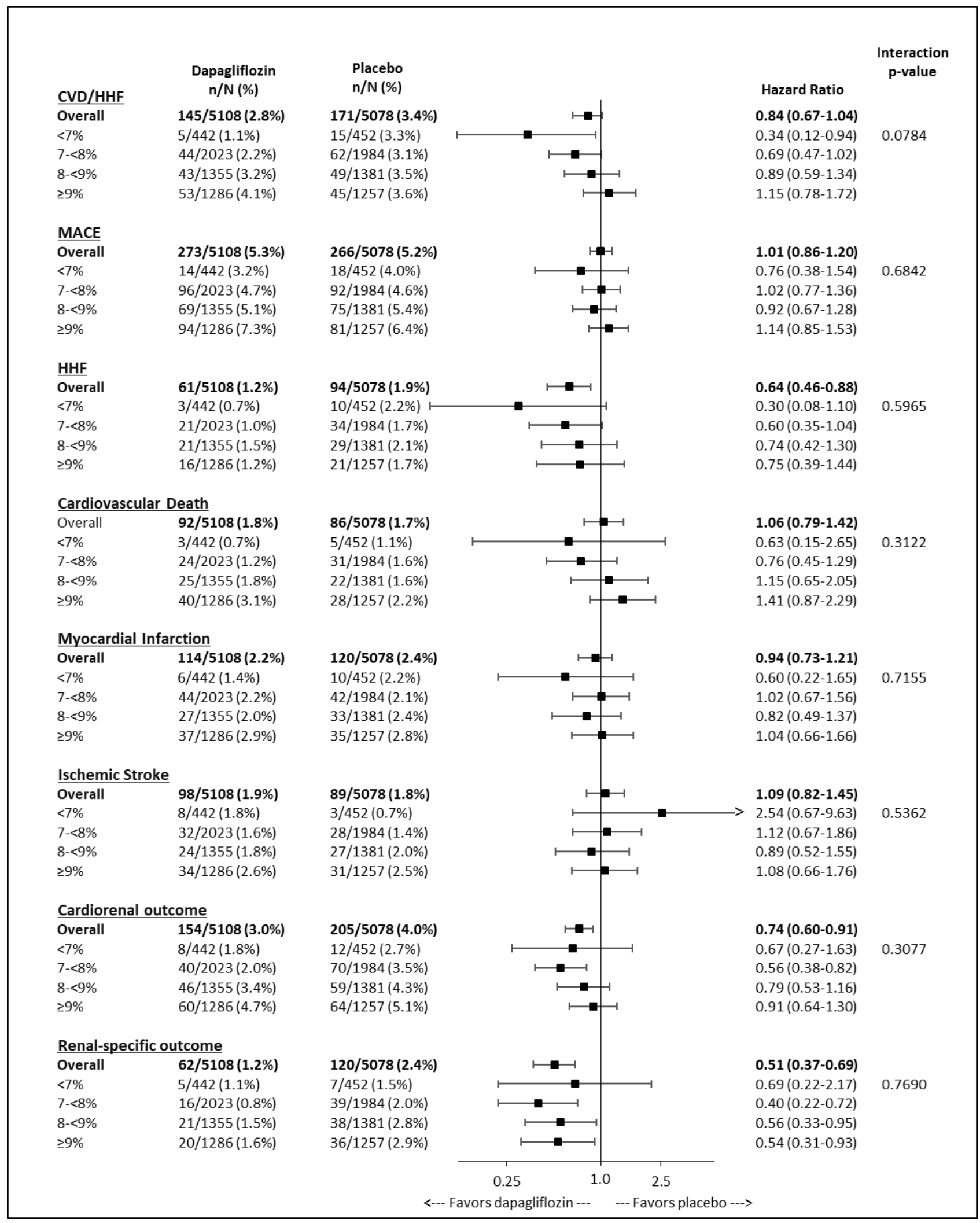




\section{Supplementary figure 4}

$\mathrm{HbA1c}$ changes with dapagliflozin vs. placebo by baseline HbA1c category

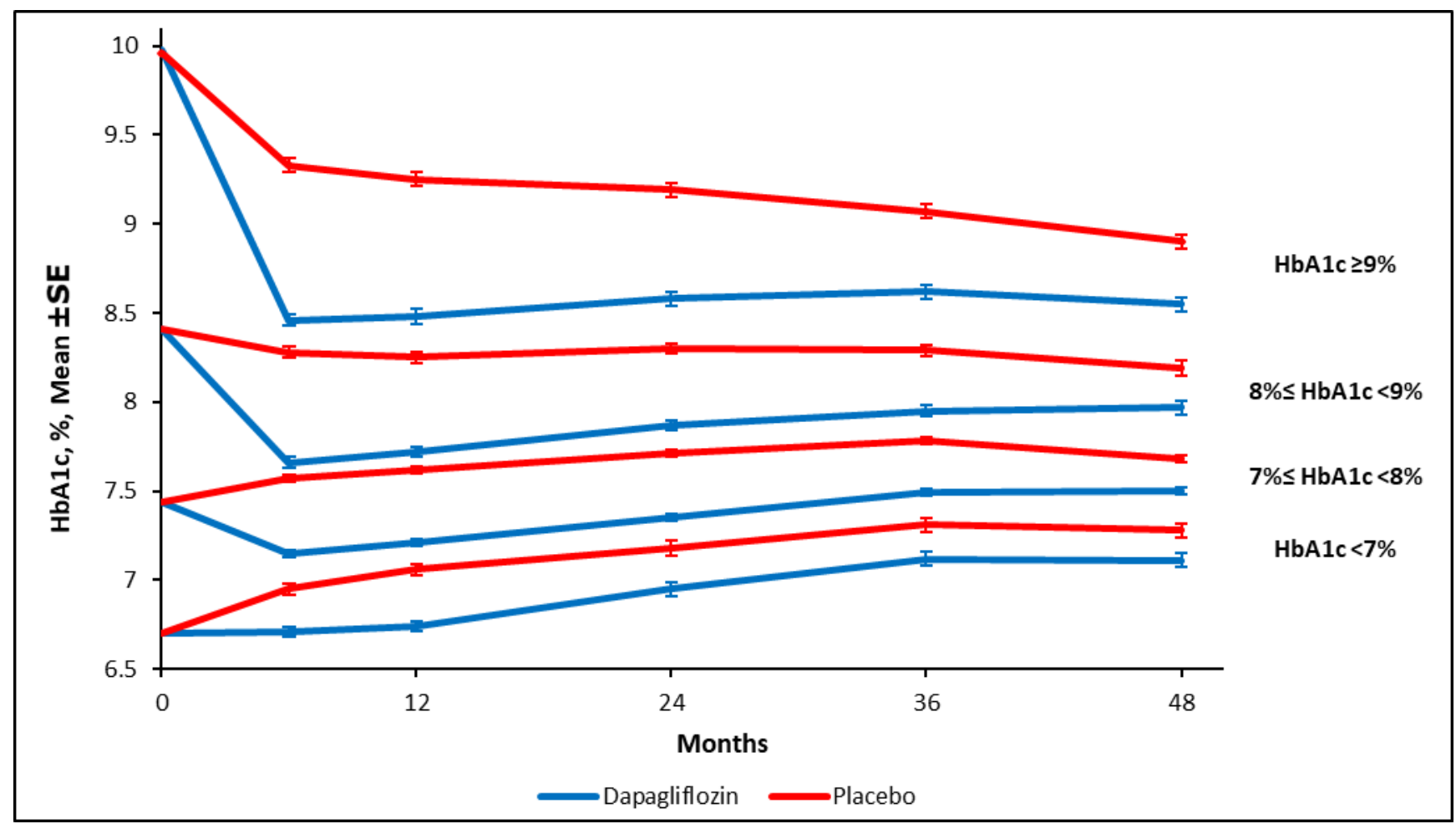

